\section{Using Experimental Petrology to Explore Exoplanet Melts and Solidi: Preliminary Implications for Habitability}

\author{
KARA BRUGMAN ${ }^{1}$, MITCHELL G. PHILLIPS ${ }^{2}$ AND \\ CHRISTY B. TILL ${ }^{3}$
}

${ }^{1}$ Carnegie Institution for Science

${ }^{2}$ USGS

${ }^{3}$ Arizona State University

Presenting Author: kara.brugman@gmail.com

Key questions driving the field of exoplanet research require an understanding of what minerals and rock types are available for surface geochemical reactions that could potentially support life. Soon we will be able to characterize exoplanet atmospheres (e.g., [1, 2, 3]) but observations of surface chemistries (i.e., crusts) remain forthcoming. Answering questions about exoplanets' compositions, interior structures, and near surface conditions with regards to their suitability for life necessitates applying methods from the field of experimental petrology.

To determine hypothetical exoplanet mantle solidi and the compositions of likely exoplanet partial mantle melts, which are the building blocks for the exoplanet's crust, we conducted anhydrous piston-cylinder experiments over 1-2 GPa and 1100$1475^{\circ} \mathrm{C}$ on two non-Earth silicate exoplanet compositions. The first hypothetical exoplanet starting composition (HEX1) was created by adjusting the undepleted Earth mantle composition to produce a molar $\mathrm{Mg} / \mathrm{Si}$ ratio of 1.23 (Earth's undepleted mantle $\mathrm{Mg} / \mathrm{Si}=1.06$ [4]). The second hypothetical exoplanet starting composition (HEX2) has a lower $\mathrm{Mg} / \mathrm{Si}$ than that of Earth $(0.82$ vs. 1.06), but a higher molar $\mathrm{Ca} / \mathrm{Al}$ ratio (1.58 vs. 1.07 [4]). HEX1 and HEX2 were not intended to directly simulate observed exoplanets.

Results indicate that a slightly higher bulk silicate $\mathrm{Mg} / \mathrm{Si}$ ratio will not result in a an exoplanet with broadly different petrological characteristics from those of Earth. However, increasing the $\mathrm{Ca} / \mathrm{Al}$ ratio has the potential to produce an exoplanet with either a molten surface (Fig. 1) or a surface devoid of volcanic activity, neither of which are favorable for habitability.

[1] Koll D. D. B. et al. (2019) ApJ 886(140), 13. [2] LustigYaeger J. (2019) $A J$ 158(1), 28. [3] Mansfield M. et al. (2019) ApJ 886(141), 11. [4] Hart S. R. and Zindler A. (1986), Chem. Geo. 57, 247-267. [5] Hirschmann M. M. (2000) $G^{3}$ 1(10), 26. [6] Putirka K. (2016) Am. Min. 101(4), 819-840.
Fig. 1

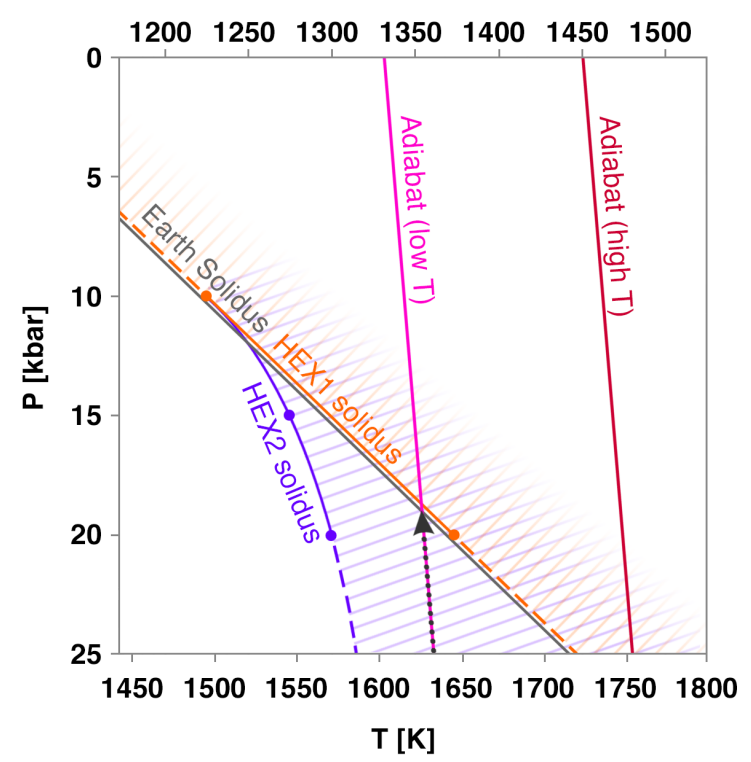

Figure 1: P-T diagram showing the locations of the HEX1 and HEX2 solidi (dashed beyond the range of experimental conditions). Earth's anhydrous undepleted mantle solidus [5] is indistinguishable from that of HEX1. The side of the solidus where melt can exist is shaded. Modern-day Earth adiabats are pinned to bracketing mantle $\mathrm{T}_{\text {pot }}$ estimates $\left(1330-1450^{\circ} \mathrm{C}\right.$ [6]). The gray arrow indicates a sample decompression melting path that intersects the HEX1 solidus but may not intersect the HEX2 solidus. 